\section{LUPUS SCIENCE\& MEDICINE}

\title{
Lupus anticoagulant, disease activity and low complement in the first trimester are predictive of pregnancy
} loss

\author{
Anil Mankee, ${ }^{1}$ Michelle Petri, ${ }^{2}$ Laurence S Magder ${ }^{3}$
}

To cite: Mankee A, Petri M, Magder LS. Lupus anticoagulant, disease activity and low complement in the first trimester are predictive of pregnancy loss. Lupus Science \& Medicine 2015;2: e000095. doi:10.1136/lupus2015-000095

Received 10 April 2015 Accepted 5 August 2015

\section{CrossMark}

\footnotetext{
${ }^{1}$ Division of Rheumatology, Icahn School of Medicine at Mount Sinai, New York, New York, USA ${ }^{2}$ Division of Rheumatology and Immunology, Johns Hopkins University School of Medicine, Baltimore, Maryland, USA

${ }^{3}$ Department of Epidemiology and Public Health, University of Maryland, Baltimore, Maryland, USA
}

Correspondence to Dr Michelle Petri; mpetri@jhmi.edu

\section{ABSTRACT}

Introduction: Multiple factors, including proteinuria, antiphospholipid syndrome, thrombocytopenia and hypertension, are predictive of pregnancy loss in systemic lupus erythematosus (SLE). In the PROMISSE study of predictors of pregnancy loss, only a battery of lupus anticoagulant tests was predictive of a composite of adverse pregnancy outcomes. We examined the predictive value of one baseline lupus anticoagulant test (dilute Russell viper venom time) with pregnancy loss in women with SLE.

Methods: From the Hopkins Lupus Cohort, there were 202 pregnancies from 175 different women after excluding twin pregnancies and pregnancies for which we did not have a first trimester assessment of lupus anticoagulant. We determined the percentage of women who had a pregnancy loss in groups defined by potential risk factors. The lupus anticoagulant was determined by dilute Russell viper venom time with appropriate mixing and confirmatory testing. Generalised estimating equations were used to calculate $p$ values, accounting for repeated pregnancies in the same woman.

Results: The age at pregnancy was $<20$ years $(2 \%)$, 20-29 (53\%), 30-39 (41\%) and >40 (3\%). 55\% were Caucasian and $34 \%$ African-American. Among those with lupus anticoagulant during the first trimester, 6/16 (38\%) experienced a pregnancy loss compared with only $16 / 186(9 \%)$ of other pregnancies ( $p=0.003)$. In addition, those with low complement or higher disease activity had a higher rate of pregnancy loss than those without ( $p=0.049$ and 0.005 , respectively). In contrast, there was no association between elevated anticardiolipin in the first trimester and pregnancy loss. Conclusions: The strongest predictor of pregnancy loss in SLE in the first trimester is the lupus anticoagulant. In addition, moderate disease activity by the physician global assessment and low complement measured in the first trimester were predictive of pregnancy loss. These data suggest that treatment of the lupus anticoagulant could be considered, even in the absence of history of pregnancy loss.

\section{INTRODUCTION}

Rates of pregnancy loss in systemic lupus erythematosus (SLE) have improved over the

\section{KEY MESSAGES}

A positive Lupus anticoagulant in the first trimester, rather than a previous positive result, is predictive of pregnancy loss.

- High disease activity as well as low complement levels during the first trimester is also predictive of pregnancy loss.

decades. Clark et $a l^{1}$ found a decrease in fetal loss rates from $40 \%$ to $17 \%$ based on a literature review from 1960 to 2000. More recent cohort studies have reported pregnancy loss rates in the range of $10-25 \% .^{2-10}$

Genetic anomalies, chromosomal abnormalities, anatomical defects and hormonal dysfunction all contribute to first trimester fetal loss in patients with SLE just as they do in the general population. ${ }^{11-14}$ In SLE, however, other risk factors for poor fetal outcomes from the first trimester to the neonatal period play a major role. We previously reported four factors at the first pregnancy visit to predict pregnancy loss: proteinuria (>500 $\mathrm{mg}$ in a $24 \mathrm{~h}$ urine collection or urine protein-to-creatinine ratio $>0.5$ ), thrombocytopenia (platelet count $<150000$ ) and antiphospholipid syndrome and hypertension (blood pressure $>140 / 90 \mathrm{~mm} \mathrm{Hg}$ ).${ }^{9}$ We have also found high lupus activity as defined by the physician global assessment score $>2$, on a $0-3$ visual analogue scale, a risk factor for fetal loss. ${ }^{10}$ Additional risk factors reported in the literature have included positive anti-dsDNA at any time during pregnancy and low complement levels in the second trimester. $^{2}$

Clowse et $a l^{9}$ in a previous study of the Hopkins Lupus Cohort in 2006, found that patients with SLE with antiphospholipid antibodies, but without secondary antiphospholipid syndrome, had the same miscarriage rate as those patients without antiphospholipid 
antibodies (12\% vs $15 \%)$. Mecacci et $a l^{5}$ in a study of 58 lupus pregnancies divided into three groups (antiphospholipid syndrome, antiphospholipid antibody positive and antiphospholipid antibody negative), found no differences in the live birth rate. In a prospective study by Lima $e t a l^{15}$ in 1995 of 108 pregnancies in patients with SLE, lupus anticoagulant did not predict fetal loss $(\mathrm{p}=0.056)$. In 1994, Derksen et $a l^{16}$ described 35 pregnancies in 25 patients with SLE and found that there was no difference in the live birth rate between those patients with antiphospholipid antibodies and those without.

In contrast to the previous findings, two studies have found increased rates of fetal loss in patients with antiphospholipid antibodies. In 2002, Moroni et al ${ }^{17}$ studied 70 pregnancies in 48 patients with lupus nephritis. In both univariate and multivariate analyses, the presence of antiphospholipid antibodies was significantly associated with increased fetal loss. There was, however, no differentiation between the presence of lupus anticoagulant and anticardiolipin antibodies. In the PROMISSE study, Lockshin et $a l^{3}$ studied pregnancies in SLE or in antiphospholipid-positive women versus control pregnancies. Adverse pregnancy outcome was defined as fetal demise after 12 weeks, neonatal death prior to discharge, preterm delivery prior to 34 weeks and small for gestational age. In the PROMISSE study, lupus anticoagulant was considered positive if it was identified by any of three tests: dilute Russell viper venom time (dRVVT), a lupus anticoagulant-sensitive partial thromboplastin time or the dilute prothrombin time. ${ }^{21}$ It was found that the lupus anticoagulant predicted adverse pregnancy outcome $(\mathrm{p}<0.0001)$. In multivariate analysis, but not in bivariate analysis, the presence of SLE conferred a relative risk of $2.16(\mathrm{p}=0.005)$.

In this paper, we report on an updated set of pregnancies from the Hopkins Lupus Cohort. Our goal was to assess the association of lupus anticoagulant detected by the dRVVT in the first trimester with the risk of pregnancy loss in patients with SLE.

\section{PATIENTS AND METHODS}

Cohort

We performed an analysis of pregnancies among patients in the Hopkins Lupus Cohort for which there was a measure of lupus anticoagulant during the first trimester. The Hopkins Lupus Cohort consists of consecutively enrolled patients with SLE who have been followed by one rheumatologist (MP) from 1987 to 2012 at the Hopkins Lupus Center. Patients were seen at 4-week to 6-week intervals during their pregnancy until delivery.

Pregnancy was confirmed by urine and serum beta human chorionic gonadotrophin tests and fetal ultrasound. Pregnancy losses were defined as any fetal death in utero. Pregnancy outcomes were obtained from obstetric records. Cohort pregnancies were excluded from the analysis if they were not singleton, if there was uncertainty about the outcome or if the patient was not assessed for lupus anticoagulant in the first trimester.

The physician global assessment score was obtained at each visit. This validated visual analogue scale assesses lupus activity (0, no activity; 1, mild lupus activity; 2, moderate lupus activity; 3 , severe lupus activity). ${ }^{18}$

Lupus anticoagulant was screened by the dRVVT and then confirmed by mixing studies and confirmatory tests. ${ }^{19}$ Anticardiolipin was determined by ELISA (Inova).

\section{Statistical analysis}

We identified subgroups of pregnancies based on their characteristics during the first trimester and compared them with respect to pregnancy loss rates. Subgroups were defined by age, ethnicity, year of conception, disease activity and serological activity. The statistical significance of each observed difference was determined using a generalised estimating equation approach to adjust for the fact that some women contributed more than one pregnancy. We performed a sensitivity analysis using only the first pregnancy for each woman and compared rates using Fisher's exact test. Analyses were performed using SAS V.9.2.

\section{RESULTS}

This analysis is based on the 202 pregnancies for which there was a first trimester assessment of lupus anticoagulant. These 202 pregnancies were from 175 women. In total, 154 women had one pregnancy, 17 had two pregnancies, 3 had three pregnancies and 1 had five pregnancies.

Fifty-three per cent of pregnancies occurred in women between the ages of 20 and 29; $41 \%$ occurred in women between the ages of 30 and 39; $3 \%$ in women over $>40$ and $2 \%$ in women younger than 20 years of age (table 1 ). The ethnic composition of our sample consisted of $55 \%$ Caucasian, 34\% African-American and $11 \%$ other ethnicity.

There were 22 pregnancy losses out of the 202 pregnancies $(11 \%)$. Of these, $12(55 \%)$ occurred within the first trimester, 9 (40\%) occurred during the second trimester and 1 (5\%) occurred during the third trimester.

There were no statistically significant differences in frequency of pregnancy losses by age group, ethnicity or year of conception (table 2), although $3 / 6$ pregnancies $(50 \%)$ among those 40 years of age or older resulted in a pregnancy loss.

First trimester lupus anticoagulant was significantly associated with an increased pregnancy loss rate ( $p=0.0035$, table 2 ). In 186 pregnancies with a negative first trimester lupus anticoagulant, the pregnancy loss rate was $9 \%$. In the 16 pregnancies with a positive lupus anticoagulant in the first trimester, there were 6 pregnancy losses $(36 \%)$. Also, 4 of the 16 pregnancies with first trimester-positive lupus anticoagulant had a history of previous thrombosis; 2 of the 6 pregnancies with pregnancy loss and 2 of the 10 without. 
Table 1 Characteristics of singleton pregnancies in the Hopkins Lupus Cohort

\begin{tabular}{|c|c|}
\hline Characteristic & $\begin{array}{l}\text { Number }(\%) \text { of } \\
\text { pregnancies }(\mathrm{N}=202)\end{array}$ \\
\hline \multicolumn{2}{|l|}{ Age (years) } \\
\hline$<20$ & $5(2 \%)$ \\
\hline $20-29$ & $108(53 \%)$ \\
\hline 30-39 & $83(41 \%)$ \\
\hline $40+$ & $6(3 \%)$ \\
\hline \multicolumn{2}{|l|}{ Ethnicity } \\
\hline Caucasian & $112(55 \%)$ \\
\hline African-American & $68(34 \%)$ \\
\hline Other & $22(11 \%)$ \\
\hline \multicolumn{2}{|l|}{ Year of conception } \\
\hline 1986-1999 & $78(38 \%)$ \\
\hline 2000-2004 & $42(21 \%)$ \\
\hline $2005+$ & $82(41 \%)$ \\
\hline \multicolumn{2}{|l|}{ Previous clinical manifestations } \\
\hline Malar rash & $(54 \%)$ \\
\hline Discoid rash & $(18 \%)$ \\
\hline Photosensitivity & $(50 \%)$ \\
\hline Oral ulcers & $(46 \%)$ \\
\hline Arthritis & $(66 \%)$ \\
\hline Serositis & (41\%) \\
\hline Seizures or psychosis & $(9 \%)$ \\
\hline \multicolumn{2}{|l|}{ Previous laboratory abnormalities } \\
\hline Antinuclear antibodies & $(97 \%)$ \\
\hline Proteinuria & (42\%) \\
\hline $\begin{array}{l}\text { Immunologic (lupus } \\
\text { anticoagulant, anticardiolipin, } \\
\text { anti-dsDNA, anti-Smith, false } \\
\text { positive rapid plasma reagin) }\end{array}$ & (82\%) \\
\hline
\end{tabular}

A history of lupus anticoagulant prior to pregnancy was not predictive of pregnancy loss (table 2). In fact, of the 25 patients with a history of lupus anticoagulant who did not have lupus anticoagulant during the first trimester, none had a pregnancy loss.

A score of $\geq 2$ on the physician global assessment (on a 0-3 visual analogue scale) during the first trimester was statistically associated with increased risk of pregnancy loss (29\% vs $8 \%, p=0.005)$. Although the numbers in some of the subgroups were not large enough to perform a complex multivariable analysis, an analysis of the association between lupus anticoagulant in the first trimester, scores of disease activity and pregnancy loss was performed. Of the 11 patients with lupus anticoagulant but without high disease activity during the first trimester, 3 (27\%) experienced a pregnancy loss. Of the three with both lupus anticoagulant and high disease activity during the first trimester, two experienced a pregnancy loss. Statistically, the association between lupus anticoagulant and pregnancy loss persisted after adjustment for high physician global assessment $(\mathrm{p}=0.013)$.

Table 2 Pregnancy loss rates by characteristics of the patients

\begin{tabular}{lc}
\hline $\begin{array}{l}\text { Patient } \\
\text { characteristic }\end{array}$ & $\begin{array}{c}\text { Proportion (\%) } \\
\text { pregnancy lo }\end{array}$ \\
\hline All & $22 / 202(11 \%)$ \\
Age (years) & \\
$\quad<30$ & $9 / 113(8 \%)$ \\
$\quad 30-39$ & $10 / 83(12 \%)$ \\
$\quad 40+$ & $3 / 6(50 \%)$ \\
Ethnicity & \\
$\quad$ Caucasian & $12 / 112(11 \%)$ \\
$\quad$ African-American & $8 / 68(12 \%)$ \\
$\quad$ Other & $2 / 22(9 \%)$ \\
Year of conception & \\
$\quad$ 186-1999 & $13 / 78(17 \%)$ \\
$2000-2004$ & $5 / 42(12 \%)$ \\
2005+ & $4 / 82(5 \%)$
\end{tabular}

Lupus anticoagulant measured in first trimester

by dilute Russell viper venom time (confirm)
Negative
$16 / 186(9 \%)$
Positive
$6 / 16(38 \%)$

History of lupus anticoagulant by dilute Russell viper venom time prior to pregnancy
No
$18 / 167(11 \%)$
Yes
$4 / 35(11 \%)$

Anticardiolipin IgG measured during first

trimester†

$\begin{array}{lr}\text { Normal } & 9 / 108(8 \%) \\ \text { High } & 0 / 7(0 \%)\end{array}$

Ever positive for anticardiolipin IgG

$\begin{array}{lc}\text { No } & 7 / 74(9 \%) \\ \text { Yes } & 15 / 128(12 \%)\end{array}$

Physician global assessment $>2$ during Value $^{*}$

pregnancy

$\begin{array}{lr}\text { No } & 15 / 180(8 \%) \\ \text { Yes } & 6 / 21(29 \%)\end{array}$

Low complement during first trimester

No $\quad 8 / 118(7 \%)$

Yes 13/83 (16\%)

Anti-dsDNA during first trimester
No
$12 / 140(9 \%)$
Yes
9/61 (15\%)

Mean prednisone dose during first trimester

$<10 \quad 12 / 146(8 \%)$

$10+\quad 9 / 55(16 \%)$

History of thrombosis prior to pregnancy

No 20/182 (11\%)

Yes $\quad 2 / 20(10 \%)$

History of miscarriage prior to pregnancy

No $12 / 132(9 \%)$

Yes $\quad 10 / 70(14 \%)$

Antiphospholipid syndromeł prior to pregnancy $\quad 0.58$

$$
\text { No } \quad 15 / 148(10 \%)
$$

Yes $\quad 7 / 54(13 \%)$

${ }^{*}$ Calculated based on a generalised estimating equation model to account for repeated pregnancies from the same woman.

†Calculated with Fisher's exact test.

fDefined as history of thrombosis or recurrent miscarriage and either history of lupus anticoagulant or elevated anticardiolipin. 
Anticardiolipin IgG levels were measured at the first pregnancy visit in 115 pregnancies. Among the seven patients with high IgG titres, none had a miscarriage. Sixty-three per cent of pregnancies had a history of positive anticardiolipin antibodies. There was no significant difference in the pregnancy loss rate between those pregnancies with or without a history of anticardiolipin IgG antibodies ( $12 \%$ vs $9 \%, \mathrm{p}=0.66)$.

Low complement levels occurred in the first trimester in 83 pregnancies $(41 \%)$. The pregnancy loss rate in this group was $16 \%$. When compared with the 118 pregnancies with normal complement levels in the first trimester, the loss rate was $7 \%(\mathrm{p}=0.049)$. A positive anti-dsDNA was not significantly associated with increased risk of pregnancy loss $(15 \%$ vs $9 \%, \mathrm{p}=0.19)$. A prednisone dose $>10 \mathrm{mg} /$ day at the first pregnancy visit was more frequent in those with pregnancy loss ( $16 \%$ vs $8 \%, \mathrm{p}=0.09)$. Rates of pregnancy loss were somewhat elevated among those with a history of thrombosis or prior miscarriage; however, these differences were not statistically significant in this small sample.

As a sensitivity analysis, we performed the same analyses using only the first pregnancy for each woman. This analysis was based on 175 women. In this analysis, we found that there was still a significant association between pregnancy loss and lupus anticoagulant in the first trimester: pregnancy losses were $5 / 13(38 \%)$ vs 20/162 (6\%) for those with and without lupus anticoagulant $(\mathrm{p}=0.0020)$. A positive association between pregnancy loss and both high disease activity and low complement was also seen in this smaller sample ( $p=0.016$ and 0.068 , respectively).

\section{DISCUSSION}

Our results demonstrate an increased risk of pregnancy loss associated with the presence of lupus anticoagulant by the dRVVT at the first trimester visit; however, a history of positive lupus anticoagulant was not associated. We also found that lupus activity defined by the physician global assessment in the first trimester was significantly associated with increased risk of pregnancy loss. Other variables including age, ethnicity, high titres of anticardiolipin antibody, use of prednisone dose as a surrogate for lupus activity and the presence of anti-dsDNA were not significantly associated with increased risk of pregnancy loss. Lupus anticoagulant remained statistically significant in multiple variable models that included disease activity and low complement.

The impact of antiphospholipid antibodies on pregnancy loss has previously been reported in this cohort. ${ }^{9}$ Clowse et al found an increased risk of total pregnancy loss in patients with SLE with antiphospholipid syndrome. The presence of either lupus anticoagulant or anticardiolipin antibody, but without the clinical criteria for classification of antiphospholipid syndrome, did not increase the risk of miscarriage. In this study, we looked separately at the contribution of lupus anticoagulant and anticardiolipin antibody to the risk of pregnancy loss. While lupus anticoagulant was strongly associated with increased pregnancy loss risk, anticardiolipin antibody was not associated with increased risk. Our current results differ from our past report in that we have now looked at each antiphospholipid antibody separately.

Our study looked at the utility of one single lupus anticoagulant test, the dRVVT, at the first pregnancy visit with one outcome (pregnancy loss). Thus, we confirm the PROMISSE finding that only the lupus anticoagulant 'matters' but extend the PROMISSE finding to the most important of the adverse pregnancy outcomes, namely pregnancy loss. Our study differed from PROMISSE in that dRVVT was done as the lupus anticoagulant assay; only first trimester results were used; we excluded PROMISSE patients and a different outcome measure (pregnancy loss) was used.

The PROMISSE study could not evaluate the contribution of high disease activity, as such patients were excluded. High disease activity assessed by physician global assessment score of $\geq 2$ in our study was significantly associated with an increased risk of pregnancy loss. Prednisone dose as a surrogate for disease activity was higher as well, but did not meet statistical significance. Clowse $e t a l,{ }^{10}$ in a previous analysis of our cohort in 2004, demonstrated a significant decrease in the live birth rate $(84 \%$ vs $57 \%$, $\mathrm{p}=0.04$ ) in pregnancies with high versus low lupus activity in the first trimester defined by the physician global assessment. Chakravarty et $a t^{22}$ defined active disease at conception as the use of $10 \mathrm{mg}$ of prednisone daily. With only six pregnancy losses in the first trimester and none in the second or third trimester, they were unable to demonstrate an increased risk of pregnancy loss. We have extended our previous work to show the independent effect of disease activity in multiple variable models.

Our study found that low complement during the first trimester was associated with an increased risk of pregnancy loss. Between 1992 and 2003, Ramos-Casals et $a l^{23}$ routinely measured complement levels in 530 female patients with SLE. They found similar rates of pregnancy loss in those patients with low complement (14\%) compared with those patients with normal complement. This is in contrast to the data published by Cortes-Hernandez $e t a l^{24}$ in 2002, in which low complement levels detected at the first visit or at 3-month intervals were significantly associated with a combination of miscarriage and stillbirth. Clowse et $a t^{2}$ previously compared low or normal complement levels and the presence or absence of anti-dsDNA in the Hopkins Lupus Cohort. Neither first trimester hypocomplementemia ( $18 \%$ vs $15 \%, \mathrm{p}=0.55)$ nor the presence of anti-dsDNA (20\% vs $13 \%, p=0.29)$ significantly increased the rate of pregnancy loss. However, low complement levels in the second trimester were associated with a significantly increased risk of fetal loss. The larger number of pregnancies in our updated analysis has allowed us to show the effect of low complement in the first trimester. 
Multicentre studies such as PROMISSE are ideal, but our study fills in three important gaps. First, one lupus anticoagulant assay, the dRVVT, with confirmatory testing, is predictive of pregnancy loss. Only the presence of the lupus anticoagulant during the pregnancy matters, as a history of lupus anticoagulant is not predictive. Thus, in clinical practice, a large battery of tests is unnecessary. Prophylactic treatment could be considered in these pregnancies. Second, high disease activity (omitted from PROMISSE) is a risk factor and control of disease activity before conception is essential to successful pregnancy outcome. Third, low complement in the first trimester is also a risk factor. In the nonpregnant patient with SLE, low complement in the absence of disease activity (serologically active, clinically quiescent) is not treated. ${ }^{25}$ Although we cannot address treatment, our study indicates the value of low complement for the prediction of pregnancy outcome.

Contributors AM contributed to writing and editing the manuscript. MP contributed to writing and editing the manuscript. LSM provided statistical analysis and editing of the manuscript.

Funding The Hopkins Lupus Cohort is supported by National Institute of Health grant AR-43727.

Competing interests None declared.

Patient consent Obtained.

Ethics approval This cohort has been approved on a yearly basis by the Johns Hopkins Institutional Review Board.

Provenance and peer review Not commissioned; externally peer reviewed.

Data sharing statement No additional data are available.

Open Access This is an Open Access article distributed in accordance with the Creative Commons Attribution Non Commercial (CC BY-NC 4.0) license, which permits others to distribute, remix, adapt, build upon this work noncommercially, and license their derivative works on different terms, provided the original work is properly cited and the use is non-commercial. See: http:// creativecommons.org/licenses/by-nc/4.0/

\section{REFERENCES}

1. Clark C, Spitzer K, Laskin C. Decrease in pregnancy loss rate in patients with systemic lupus erythematosus over a 40 year period. J Rheumatol 2005;32:1709-12.

2. Clowse ME, Magder LS, Petri M. The clinical utility of measuring complement and anti-dsDNA antibodies during pregnancy in patients with systemic lupus erythematosus. J Rheumatol 2011;38:1012-16.

3. Lockshin MD, Kim M, Laskin CA et al. Prediction of adverse pregnancy outcome by the presence of lupus anticoagulant, but not anticardiolipin antibody, in patients with antiphospholipid antibodies. Arthritis Rheum 2012;64:2311-18.
4. Yuen SY, Krizova A, Ouimet JM, et al. Pregnancy outcome in systemic lupus erythematosus is improving: Results from a case control study and literature review. Open Rheumatol J 2008;2:89-98.

5. Mecacci F, Bianchi B, Pieralli A, et al. Pregnancy outcome in systemic lupus erythematosus complicated by antiphospholipid antibodies. Rheumatology (Oxford) 2009;48:246-9.

6. Brucato A, Doria A, Frassi M, et al. Pregnancy outcome in 100 women with autoimmune diseases and anti-Ro/SSA antibodies: a prospective controlled study. Lupus 2002;11:716-21.

7. Wong $\mathrm{CH}$, Chen TL, Lee CS, et al. Outcome of pregnancy in patients with systemic lupus erythematosus. Taiwan J Obstet Gynecol 2006;45:120-3.

8. Andrade R, Sanchez ML, Alarcon GS, et al. Adverse pregnancy outcomes in women with systemic lupus erythematosus from a multiethnic US cohort: LUMINA (LVI). Clin Exp Rheumatol 2008;26:268-74.

9. Clowse ME, Magder LS, Witter F, et al. Early risk factors for pregnancy loss in lupus. Obstet Gynecol 2006;107:293-9.

10. Clowse ME, Magder LS, Witter F, et al. The impact of increased lupus activity on obstetric outcomes. Arthritis Rheum 2005;52:514-21.

11. Lathi RB, Gray Hazard FK, Heerema-McKenney A, et al. First trimester miscarriage evaluation. Semin Reprod Med 2011;29:463-9.

12. Suzumori N, Sugiura-Ogasawara M. Genetic factors as a cause of miscarriage. Curr Med Chem 2010;17:3431-7.

13. Lebedev I. Mosaic aneuploidy in early fetal losses. Cytogenet Genome Res 2011;133:169-83.

14. Porter TF, Scott JR. Evidence-based care of recurrent miscarriage Best Pract Res Clin Obstet Gynaecol 2005;19:85-101.

15. Lima F, Buchanan N, Khamashta MA, et al. Obstetric outcomes in systemic lupus erythematosus. Semin Arthritis Rheum 1995;25:184-92.

16. Derksen RH, Bruinse HW, de Groot PG, et al. Pregnancy in systemic lupus erythematosus: a prospective study. Lupus 1994:3:149-55.

17. Moroni G, Quaglini S, Banfi G, et al. Pregnancy in lupus nephritis. Am J Kidney Dis 2002;40:713-20.

18. Petri M, Genovese M, Engle E, et al. Definition, incidence and clinical description of flare in systemic lupus erythematosus. Arthritis Rheum 1991;34:937-44.

19. Petri M, Nelson L, Weimer F, et al. The automated modified Russell viper venom time test for the lupus anticoagulant. $J$ Rheumatol 1991:18:1823-5.

20. Pengo V, Tripodi A, Reber G, et al. Update of the guidelines for lupus anticoagulant detection. J Thromb Haemost 2009;7: 1737-40.

21. Chakravarty EF, Colón I, Langen ES, et al. Factors that predict prematurity and preeclampsia in pregnancies that are complicated by systemic lupus erythematosus. Am J Obs Gynecol 2005;192:1897-904.

22. Ramos-Casals M, Campoamor MT, Chamorro A, et al. Hypocomplementemia in systemic lupus erythematosus and primary antiphospholipid syndrome: prevalence and clinical significance in 667 patients. Lupus 2004:13:777-83.

23. Cortés-Hernández J, Ordi-Ros J, Paredes F, et al. Clinical predictors of fetal and maternal outcome in systemic lupus erythematosus: a prospective study of 103 pregnancies. Rheumatology (Oxford) 2002;41:643-50.

24. Steiman AJ, Gladman DD, Ibañez D, et al. Outcomes in patients with systemic lupus erythematosus with and without a prolonged serologically active clinically quiescent period. Arthritis Care Res (Hoboken) 2012;64:511-18. 